\section{Military Technical College Kobry El-Kobbah, Cairo, Egypt}

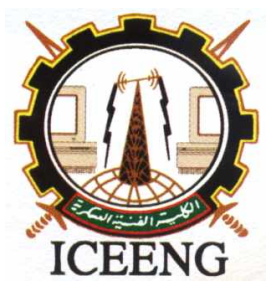

\section{$6^{\text {th }}$ International Conference on Electrical Engineering ICEENG 2008}

\title{
Modeling and simulation of pulsed Nd:YAG laser system
}

\author{
A. A. Mohammed* \\ S. S. A. Ghoniemy **
}

\section{$\underline{\text { Abstract }}$}

In this paper, we present a pulsed Nd:YAG laser model that accurately predicts the dynamic operations of a passively Q-switched Nd:YAG lasers. We introduce a new implementation technique for the proposed $\mathrm{Nd}$ :YAG laser model that provides a compelling incentive for the performance assessment and enhancement for the design of pulsed Nd:YAG lasers using SIMULINK CAD tool. The implemented laser model enables the simulation of the dynamic characteristics of a passively Q-switched Nd:YAG lasers such as carriers, photons, and optical power transient response. The proposed simulation technique is used for predicting the design parameters of practical Nd:YAG lasers. The simulation model also provides a possibility of optimizing the design in regard to optimum output coupler reflectivity, pump power and mirror spacing. Important laser characteristics, such as relaxation-oscillation peak frequency, maximum and minimum repetition rates, delay time and possibly more may be determined using the implemented model.

Comparison of experimental and simulation results of the dynamic operation is used to verify the accuracy of the proposed model for designing Nd:YAG laser systems. The measured and published results, such as pumping energy, repetition rate, and output optical power/energy are in good agreement with the simulation results.

\section{$\underline{\text { Keywords }}$}

Pulsed Nd:YAG laser modeling, Pulsed Nd:YAG laser simulation, passively Q-switched solid-state lasers, flash lamp pumped lasers.

* Optoelectronic Dep., MTC, Cairo, Egypt. Email: adelmtc2@yahoo.com

** Optoelectronic Dep. Chair, MTC, Cairo, Egypt. Email:ghoniemy@ sce.carleton.ca 


\section{Introduction}

Solid-state lasers remain an attractive area of research because of several applications in different fields and their unique capabilities such as lower threshold, higher gain, size customization for a given energy, its ability to operated at relatively high repetition rates (up to $\mathrm{kHz}$ ) and its operational stability [1]. These capabilities have opened up many attractive new areas for solid state laser research. Nd:YAG media is the most commonly used media of solid-state lasers in many fields. Its popularity has come from its wide range of output powers and the versatile output shapes available from a $\mathrm{cw}$ and a pulsed modes [2,3]. Many research efforts directed to study the performance of cw-pumped systems have been reported in details in $[4,5]$. Mathematical models of a pulse-pumped $\mathrm{Nd}$ :YAG laser have been reported in a few research $[5,6]$. However these models provided a good methodology to predict the intrinsic loss and the losses for components added to the laser due to the increase in threshold, they showed a lack in providing a comprehensive modeling that is able to predict the dynamic behavior of laser under different conditions and varying parameters. The operation of passive Q-switch has been studied in depth $[7,8,9]$ as well as the Q-switched systems optimization as in [8, $10,11]$ but neither these are complete nor comprehensive system models. They studied the Q-switch as a separate element. The design of a flashlamp power supply for pumping solid-state lasers was presented in $[12,13]$. Compactness, losses reduction efficiency enhancement of the pump source power supply and the pump source pulse shape were studied in many researches such as in $[2,3,14,15]$. Again each subsystem was individually studied. Pump cavity design has been introduced in details in $[4,5]$ and the improvement of the resonator design to enhance the laser beam quality was reported in [16]. Again all these research efforts did not provide a complete and comprehensive models for Nd:YAG laser systems. In this study, a complete and comprehensive pulsed $\mathrm{Nd}$ :YAG laser source model is introduced. A mathematical model describes the dynamic behavior of a pulsed Nd:YAG laser was implemented and simulated using SIMULINK tool provided in Matlab6.5. The implemented model is used for the design of the system including the choice of the pump source and design of its power supply, design of the optical resonator, design of the pump cavity, design and optimization of the passive Q-switch. It also used for the system optimization to provide the optimum output coupler reflectivity, pump power and mirror spacing. Good agreements between the calculated and simulated results are shown. This paper presents a pulsed Nd:YAG laser model that accurately predicts the dynamic operations of a passively Q-switched $\mathrm{Nd}$ :YAG lasers. In section 2, a three coupled differential equations is used to describe the time behavior of a 4- level solid-state lasers. In section 3, this model is implemented using MATLAB SIMULINK and the time evolution of the solid-state laser was obtained. Estimation of the threshold pump energy, description and simulation of the pumping unit and power spectrum of the laser output is also presented in section 3 . In section 4, a model for a passive Q-switch is added to the optical path in side the resonator and the pulse energy is obtained. System optimization with respect to optical 
cavity length, output coupler reflectance and saurable absorber initial transmission to maximize the laser pulse energy are presented and discussed in section 5.

\section{Solid-State Laser Modeling}

Many techniques have been reported for modeling solid-state lasers, from different points of view such as that based on the laser physics, quantum mechanics, and quantum electronics $[4,5,19]$. However these models are deeply investigated the interactions and behaviors of solid-state lasers; they are not suitable to be easily implemented in a comprehensive simulation tool. For simulating the dynamic behavior of these systems taking into account the possibility of parameters variations, one of the best techniques is the rate equation model. There are many forms of these equations, in this paper the rate equations model presented in [4] is enhanced and implemented using SIMULINK. This model consists of a three coupled differential equations and used to describe the time behavior of a 4- level solid-state lasers and given by,

$$
\begin{aligned}
& \frac{d n_{2}}{d t}=-n_{2} \sigma \phi c-\frac{n_{2}}{\tau_{f}}+W_{p}\left(n_{o}-n_{2}\right) \\
& \frac{d n_{1}}{d t}=\left(n_{2}-\frac{g_{2}}{g_{1}} n_{1}\right) \sigma \phi c+\frac{n_{2}}{\tau_{f}}-\frac{n_{1}}{\tau_{10}} \\
& \frac{d \phi}{d t}=c \phi \sigma\left(n_{2}-\frac{g_{2}}{g_{1}} n_{1}\right)-\frac{\phi}{\tau_{c}}
\end{aligned}
$$

In which $\mathrm{n}_{2}$ is the population density of the upper laser level, $\mathrm{n}_{1}$ is the population density of the lower laser level, $\Phi$ is the photon density within the laser resonator, $\tau_{\mathrm{f}}$ is the upper laser level life time, $\sigma$ is the stimulated emission cross section area, $\mathrm{c}$ is the velocity of light in vacuum, $\mathrm{n}_{\mathrm{o}}$ is the total number of atoms per unit volume, $\tau_{10}$ is the relaxation time between lower laser level and the ground level, $\tau_{\mathrm{c}}$ is the decay time for photons in the optical resonator, $\left(g_{2} / g_{1}\right)$ is the degeneracy factor and $W_{p}$ is the pump rate.

\section{Simulation Model}

\subsection{Dynamic characteristics and time evolution}

We started this research by implementing this model using MATLAB SIMULINK. Different numerical techniques were used to solve the presented set of the differential equations but most of them were very slow and did not able to predict the required output. The best solving method we found was ode23s (stiff/Mod. Rosen Brock). Using this numerical technique the time evolution of the solid-state laser was obtained as shown in figures 1 and 2. 


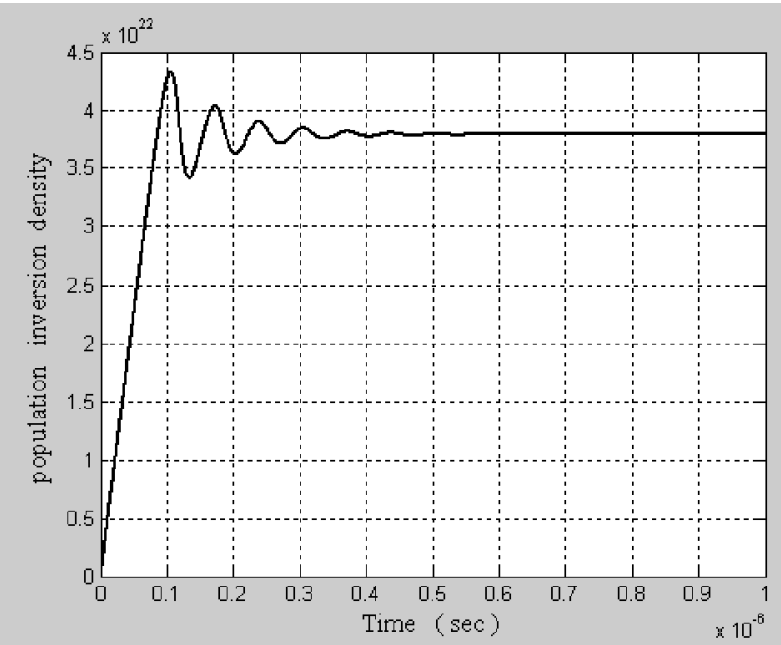

Figure 1 Time evolution of carrier density

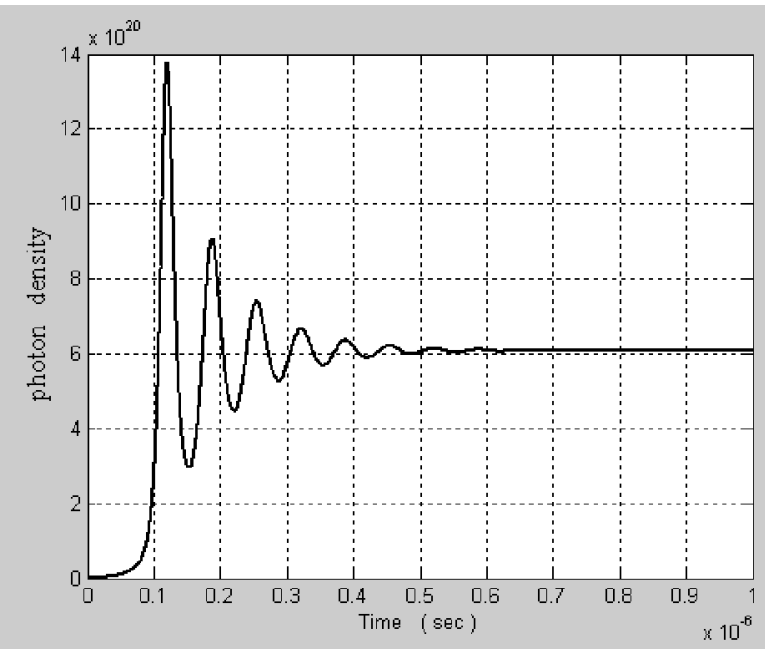

Figure 2 Time evolution of photon density

In order to properly characterize the performance of the constructed laser system the first key parameter must be known is the resonator loss L. these losses arises from scattering within the gain medium, scattering and absorption due to the mirror coating, diffraction around the edges of the internal optics, etc. Losses can be measured using two methods, the first method involves the use of output coupler of different reflectivities and then finding the threshold pump input power using different couplers [17], this method is called the Findlay resonator method. The second method directly measures the transmitted or reflected light from Nd:YAG. The Findlay resonator method for measuring resonator losses is adapted and used in this paper. The analytical relationship between pump threshold power and output mirror reflectivity is presented in [4] and given by,

$-\ln \left(R_{1}\right)=2 K E_{t h}-L$

In which $\mathrm{L}$ is the resonator combined loss, $\mathrm{k}$ is the pumping coefficient, $\mathrm{E}_{\mathrm{th}}$ is the threshold pump energy, and $R_{1}$ is the output coupler reflectance. Figure 3 shows the relation between the logarithmic output coupler reflectance and the threshold pump energy. The intersection with the y-axis represents the system combined loss L. Analyzing this figure, a slope of $2 K=0.04 \mathrm{~J}^{-1}$ is determined and hence, a loss $\mathrm{L}=0.177$ is identified. This leads to a small signal gain $\mathrm{g}_{0}=0.12 \mathrm{~cm}^{-1}$ and single pass gain $\mathrm{G}_{\mathrm{o}}=3.74$. For the purpose of practical applications these parameters enable us to calculate the threshold energy for the flash lamp required to obtain output energy of 6 $\mathrm{J} /$ pulse and found to be $\mathrm{E}_{\mathrm{th}}=31 \mathrm{~J}$. Any value of pumping energy above this value makes the system to begin lasing. Using the simulated model we determine the required pumping energy to be $E_{i n}=600 \mathrm{~J}$ for the specified output energy of $6 \mathrm{~J}$ which is corresponding to a slope efficiency of 0.012. From the threshold gain coefficient and the threshold energy density and using the model, the dimension of the laser rod is found to be $11 \mathrm{~cm}$ in length and $8 \mathrm{~mm}$ in diameter. These results were calculated based on the 
values of the system losses, threshold inversion and the small signal gain coefficient. The model was used first by applying a ramp input to obtain a graph similar to figures 1 and 2; this was to ensure that the designed and predicted parameters are right and consistent and provide the known laser dynamic behaviors.

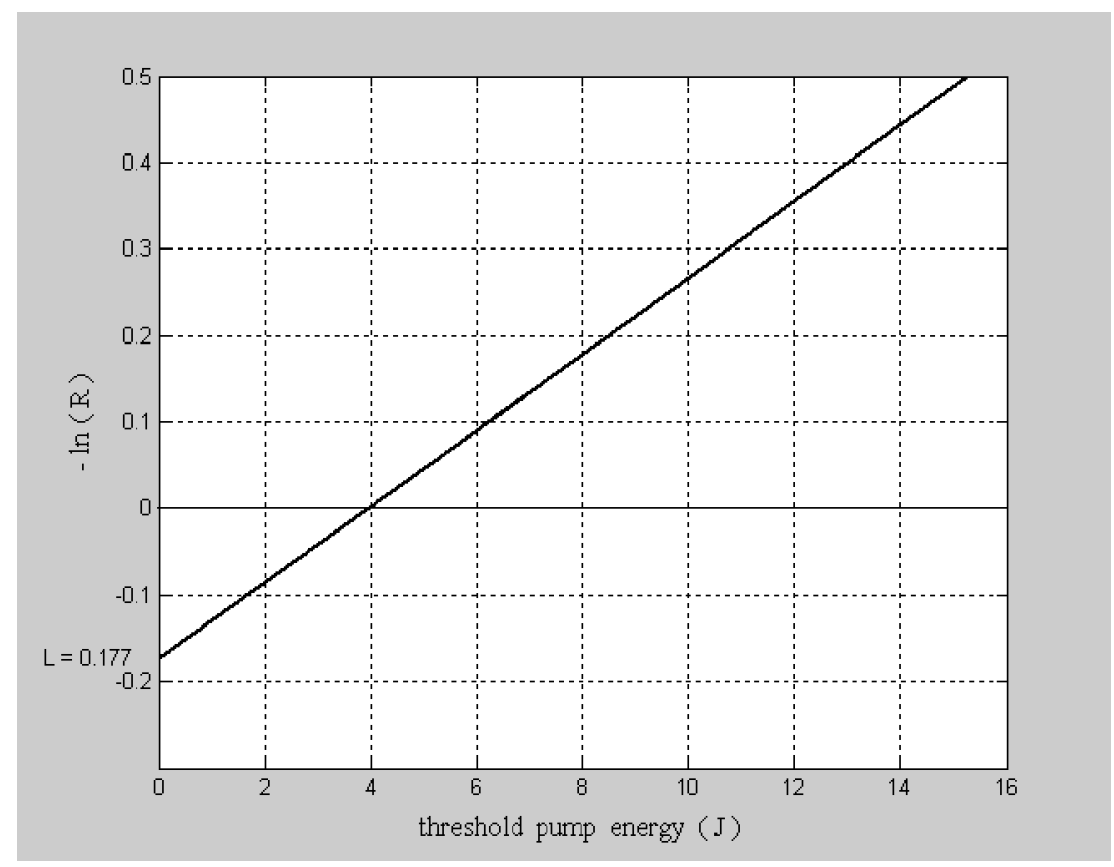

Figure 3 Logarithmic output coupler reflectance as a function of the threshold pump energy. The intersection with the y-axis represents the system combined loss.

\subsection{Pumping Unit}

\subsubsection{Flash lamp}

Development of an efficient pumping source is important in obtaining a high power and efficient Neodymium Laser. Desirable characteristics of an optical pumping source are high conversion efficiency, a good overlap of the emission spectrum with an effective absorption spectrum of the laser material, sufficiently intense irradiance, and high power operation. To obtain high pumping efficiency it is better to use a flashlamp that matches the laser rod dimensions so the required flash lamp parameters are determined using the simulation model and found to be with an arc length of $11 \mathrm{~cm}$ and bore diameter of 8 $\mathrm{mm}[4,5,18]$.

\subsubsection{Power Supply}


Design and analysis of pulsed light sources was described by a lot of authors [2-6]. High voltage circuits simulators are not available, so the flashlamp power supply was modeled and simulated using MATLAB SIMULINK. The mathematical model of the pumping circuit was used to show the behavior of the flashlamp as shown in figure 4 . Flash lamps are usually operated by an inductor L capacitor C network. The network stores the discharge energy and delivers it to the flash lamp. The pulse forming network (PFN) must correspond to the critically damped case $(\alpha=0.8)$ assuring stable flash lamp operation $[4,18]$. To obtain the required output energy, a flash lamp power supply was designed and implemented comprehensively with the intrinsic laser model and used to predict the total capacitance and found to be $285 \mu \mathrm{F}$ and the total inductance to be $318 \mu \mathrm{H}$, charging voltage to be $2 \mathrm{kV}$ and a triggering voltage to be $20 \mathrm{kV}$. Using the same model we are able to determine the input pulse energy and the pulse width and found to be $\approx 600 \mathrm{~J}$ pulse energy and pulse duration of $900 \mu \mathrm{sec}$ as shown in figure 4 . By estimating the delay time before the onset of lasing the maximum repetition rate of the system is also determined approximately to be $9 \mathrm{~Hz}$.

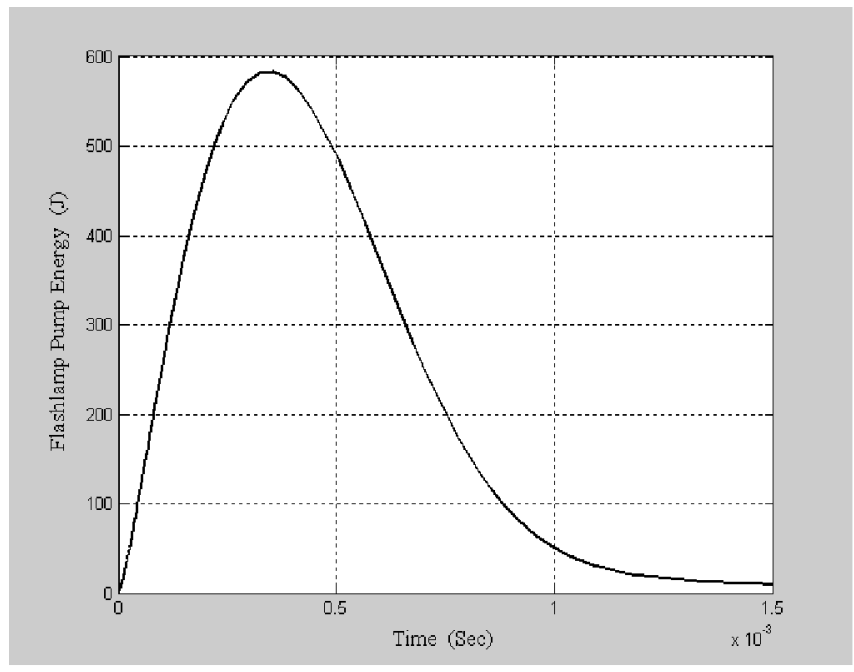

Figure 4 Flashlamp pulse energy

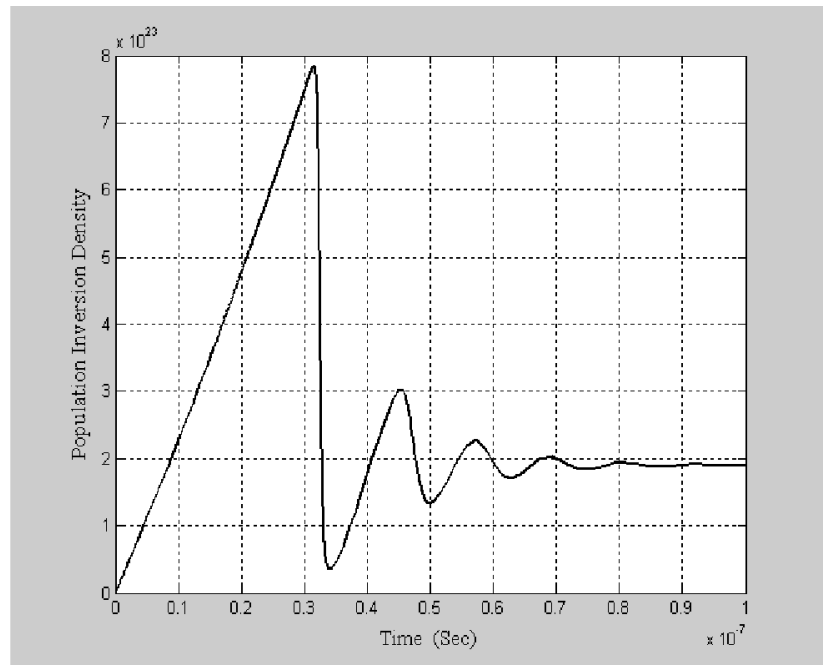

Figure 5 Population inversion density after applying 600J pump pulse

This pulse was applied as an input for the laser model. Using this model the population inversion density, photon density and the output laser energy are shown in figures 5,6,7 and 8. 


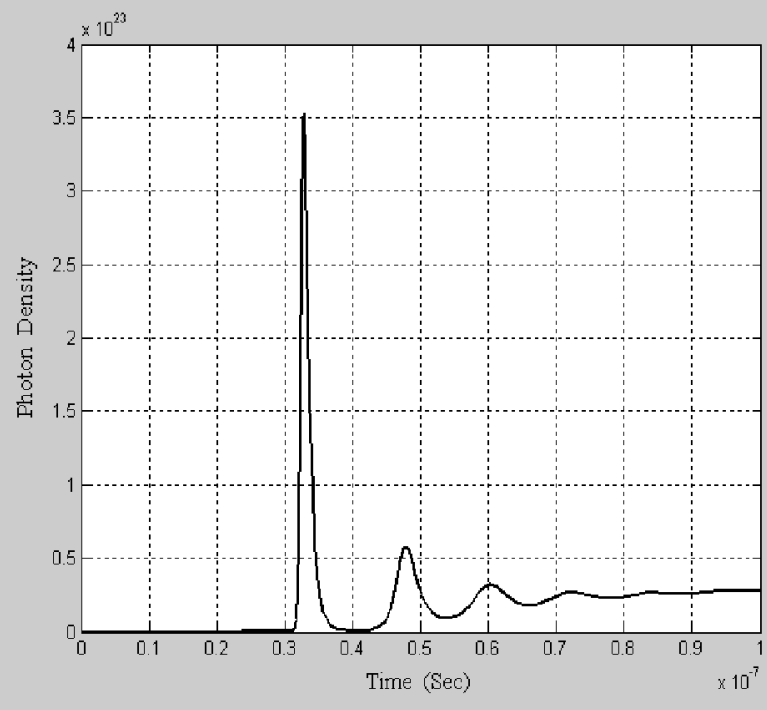

Figure 6 Time evolution of photon density after applying 600J pump pulse

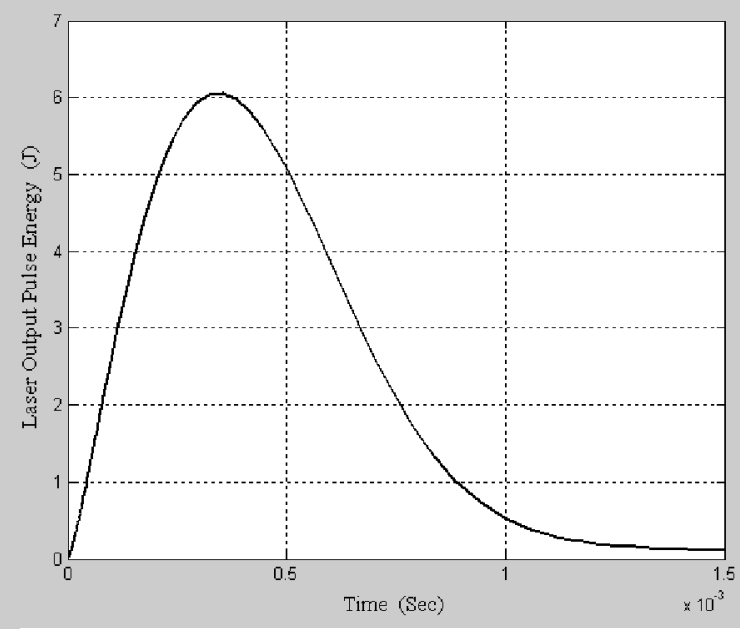

Figure 8 Laser output pulse energy after applying $600 \mathrm{~J}$ pump

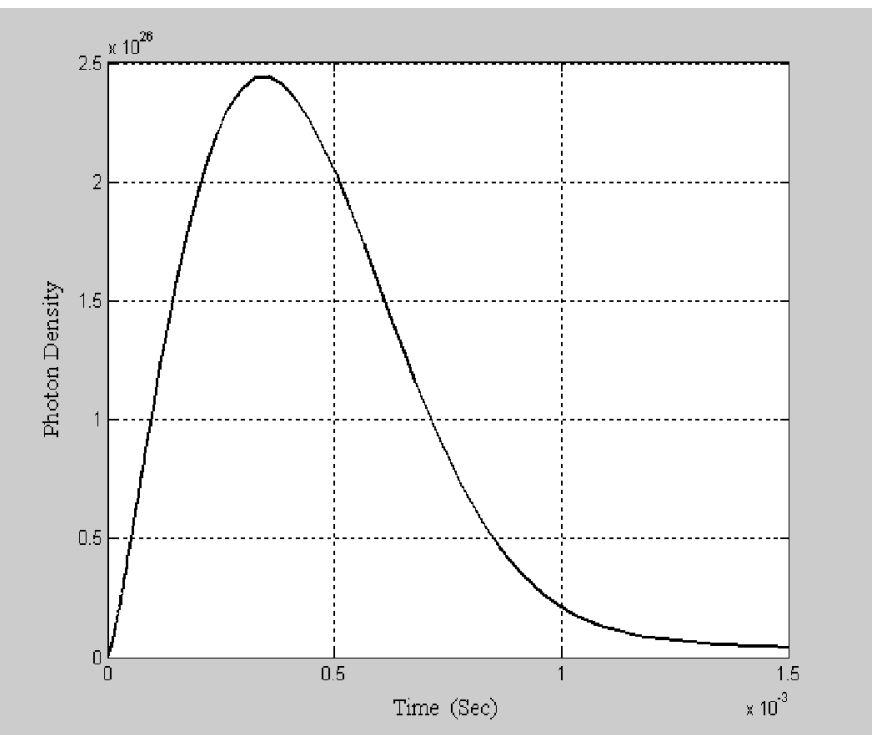

Figure7 Photon density at steady state after applying 600J pump pulse

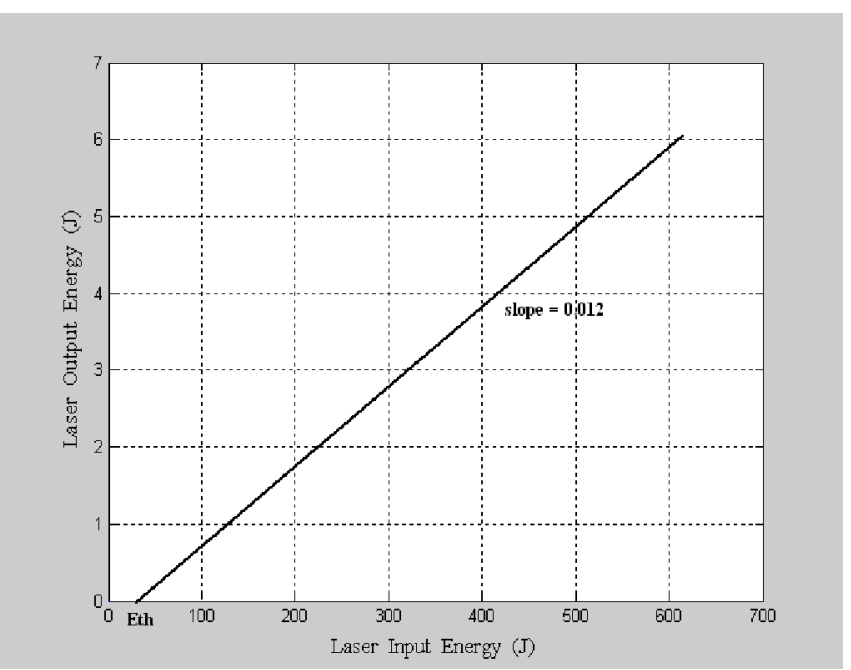

Figure 9 Output energy against pump energy

Plotting the output energy against input energy as shown in figure 9, giving threshold pump energy of $31 \mathrm{~J}$ and a slope efficiency of 0.012 for $30 \%$ output coupler reflectance. More visualization capabilities are added to the model to be able to investigate the laser characteristics in the frequency domain. In this section, we present a simulation technique which is equivalent to the OSA. This technique enables us to predict the power spectrum as shown in figure 10. 


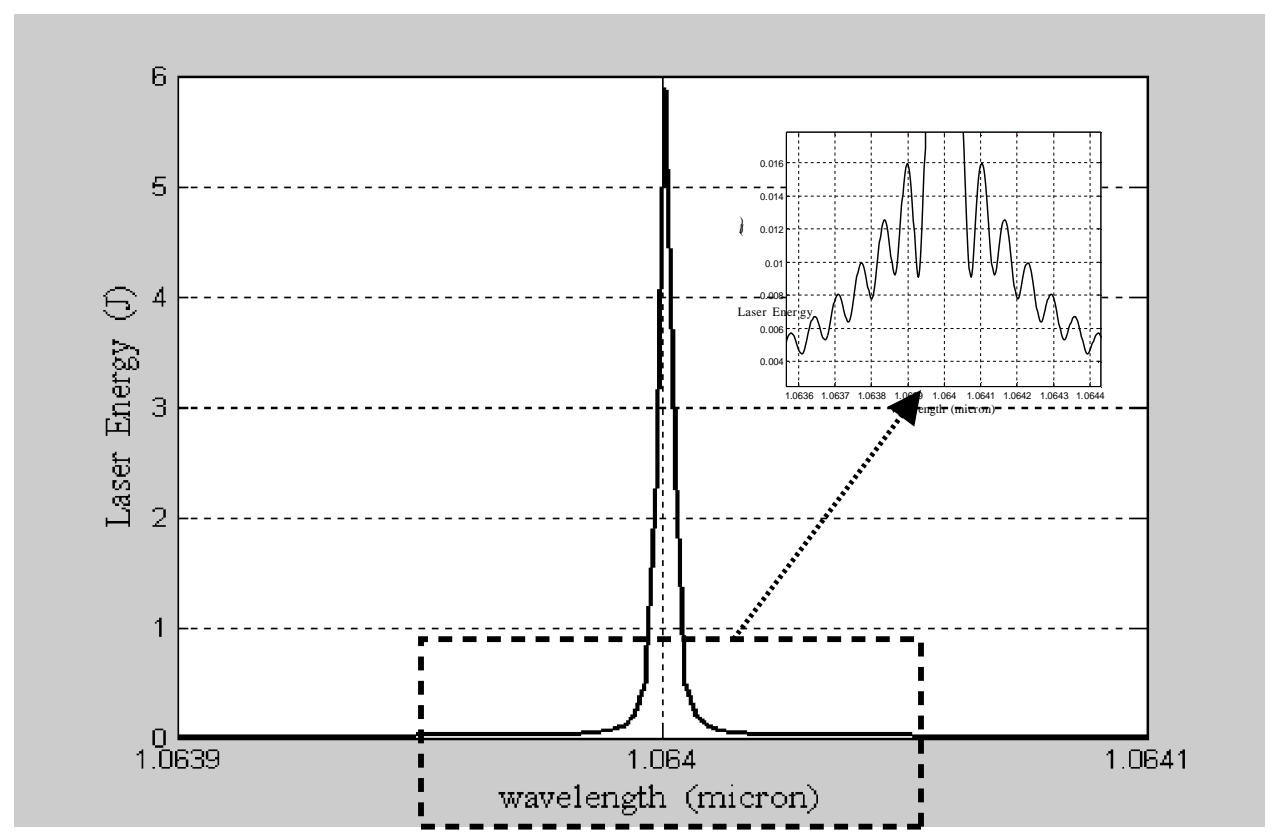

Figure 10 Power spectrum of the laser output

\section{Q-Switched System}

Passive and active Q-switches are introduced in the field of pulsed laser system design to control the output energy, to avoid large geometrical cavity dimensions and complicated power supply. Passive Q-switches are always is preferred since they do not require high voltage, fast electro-optic driver or if modulator. From the practical point of view it provides simple design, compactness and low cost systems. On the other hand, the major disadvantages of a passive Q-switch are the lack of a precise external trigger capability and the lower output compared to the active electro-optic and acoustooptic Q-switches [4]. In this research, we adopted and used the $\mathrm{Cr}^{4+}: \mathrm{YAG}$ crystal as a passive Q-switch, since the $\mathrm{Cr}^{4+}$ ions provide the high absorption cross-section of the laser wavelength and the YAG crystal provides the desirable chemical, thermal and mechanical properties required for long life operation.

To enhance the performance of our proposed simulation model we added the model of passive Q-switch in the optical path inside the resonator. Many researchers discussed the mathematical model of pasively Q-switched lasers such as in $[4,7,8,9,10,11,20]$. In this paper a generalized model for passively Q-switched lasers is used. In this model, four coupled rate equations that describe the dynamic operation of the system are solved using SIMULINK. The gain medium is a Nd:YAG cylindrical laser rod of dimensions 8 $\mathrm{mm} \times 110 \mathrm{~mm}$. Both gain medium and passive Q-switch are antireflection (AR) coated at $1.064 \mu \mathrm{m}$. All the resonator surfaces of the rod, Q -switch, and mirrors are flat, output coupler reflectance is $50 \%$ and the mirror-to-mirror distance is $15 \mathrm{~cm}$; the Cr:YAG crystal thickness , $1_{\mathrm{s}}$, is $2.5 \mathrm{~mm}$ and its diameter is $12.7 \mathrm{~mm}$ while its small signal transmission , $\mathrm{T}_{\mathrm{o}}$, is $50 \%$. Also the used saturable absorber crystal parameters as given in 
[10] and [20] are used: excited state absorption cross section $\sigma_{\mathrm{es}}=2.2 \times 10^{-19} \mathrm{~cm}^{2}$ and ground state absorption cross section $\sigma_{\mathrm{gs}}=8.7 \times 10^{-19} \mathrm{~cm}^{2}$. The concentration, $\mathrm{n}_{\mathrm{o}}$, is calculated to be $3.187 \times 10^{24} \mathrm{~m}^{-3}$ and the initial inversion of the gain medium , $\mathrm{n}_{\mathrm{i}}$, is $2 \times 10^{23} / \mathrm{m}^{3}$. Figures 11 and 12 show the simulation results of the laser system after adding the Q-switch model and illustrate the development of the Q-switched pulse.

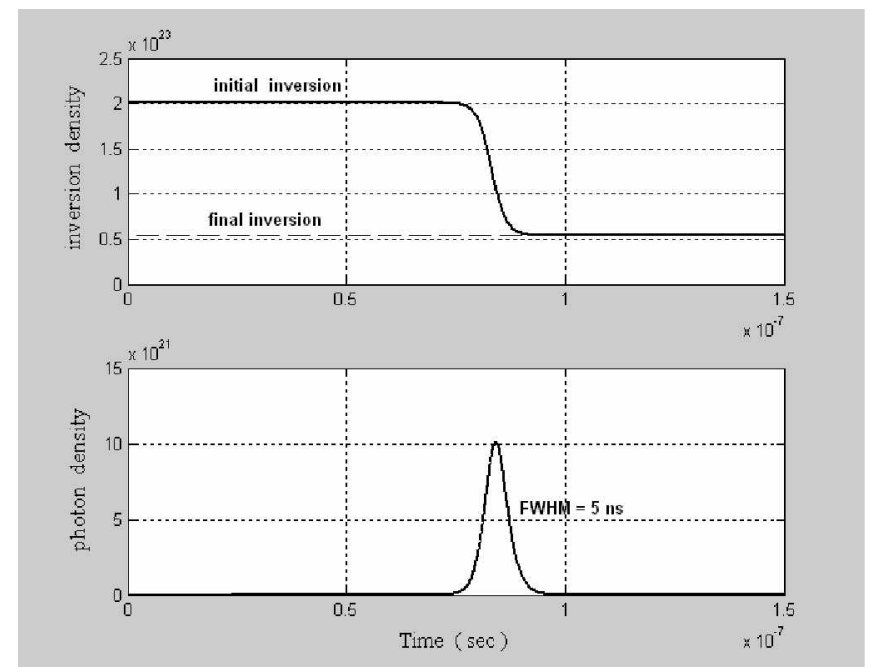

Figure 11 Time evolution of inversion and photon densities after adding the Q-switch.

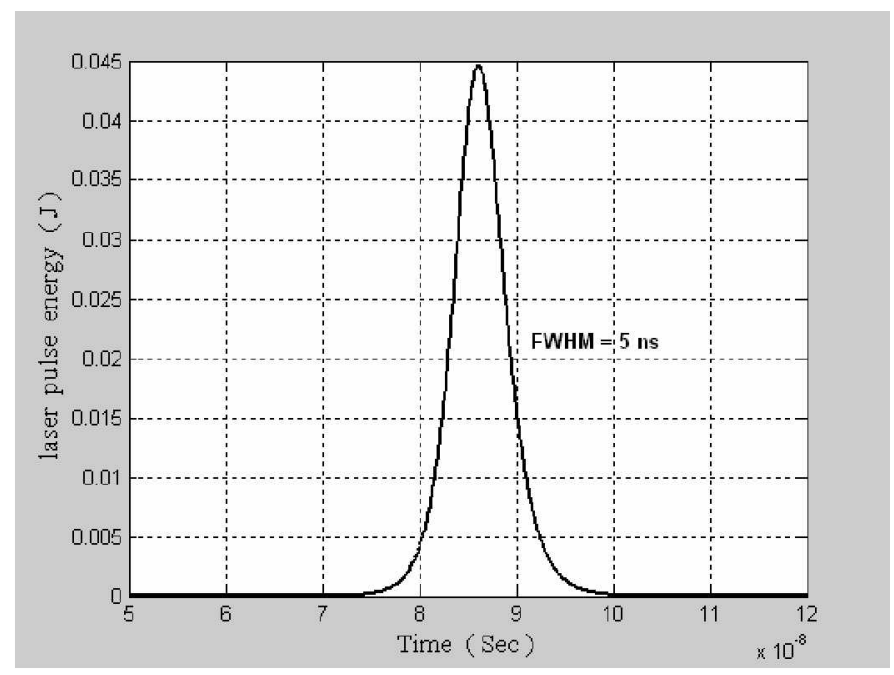

Figure 12 Development of a $Q$-switched pulse

Figure 12 shows that the output energy is $45 \mathrm{~mJ}$, FWHM pulse width is $5 \mathrm{nsec}$, and hence the peak power is approximately $9 \mathrm{MW}$. These results are in good agreement with the measured and published results in $[9,10,11]$. More enhancements for the purpose of robustness of the laser system modeling and simulation we added an optimization technique that enables the designer to variate the parameters and analyze the results or customize the parameters that meet the requirements. For the purpose of testing this technique we changed the saturable absorber initial transmission and showed its effect on the laser output pulse energy. We noticed that, using a low saturable absorber initial transmission will increase the pulse energy and vice versa. Using the proposed simulator we used a saturable absorber crystal with different initial transmissions. As an example, a saturable absorber with initial transmission of $30 \%$ and output coupler reflectance of $30 \%$, this increases the Q-switched pulse energy to $420 \mathrm{~mJ}$ and the pulse width is reduced to be $1.1 \mathrm{nsec}$ compared to the case when saturable absorber initial transmission of $50 \%$ and output coupler reflectance of $50 \%$ were used. The results of this simulation are presented in figures 13 and 14. Figure 13 shows the change of the ground state, excited state population density of the absorber and the upper laser level population inversion with time. Figure 14 presents the Q-switched pulse from which we conclude that the peak pulse energy is $420 \mathrm{~mJ}$ and the pulse width is 1.1 nsec. Taking together, figures 13 and 14 we concluded that the Q-switched laser output pulse reaches its peak value at the threshold inversion. 


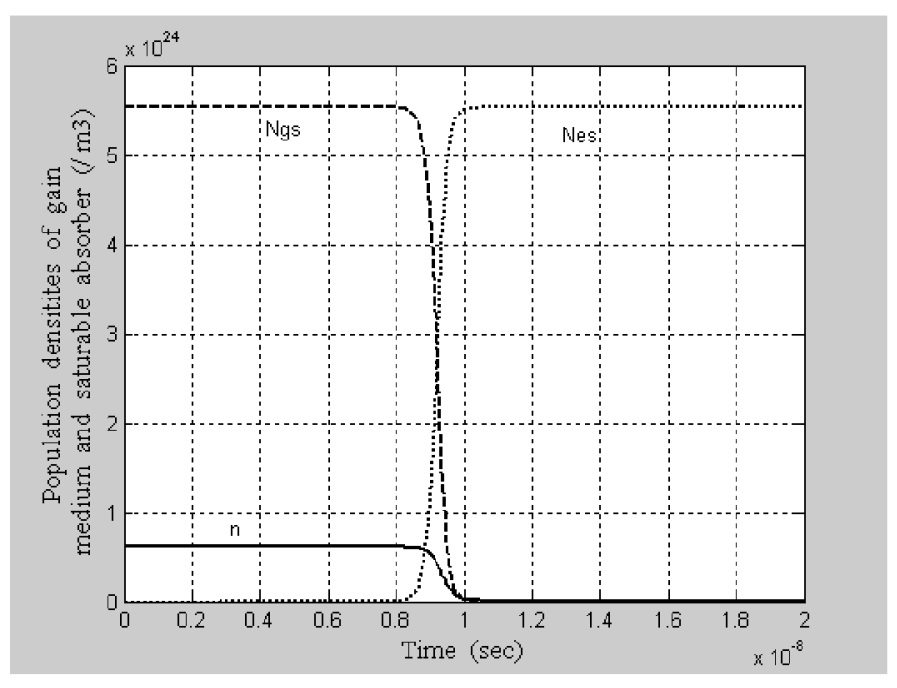

Figure 13 Change of the ground state, the excited state population density of the absorber and the upper laser level population inversion with time

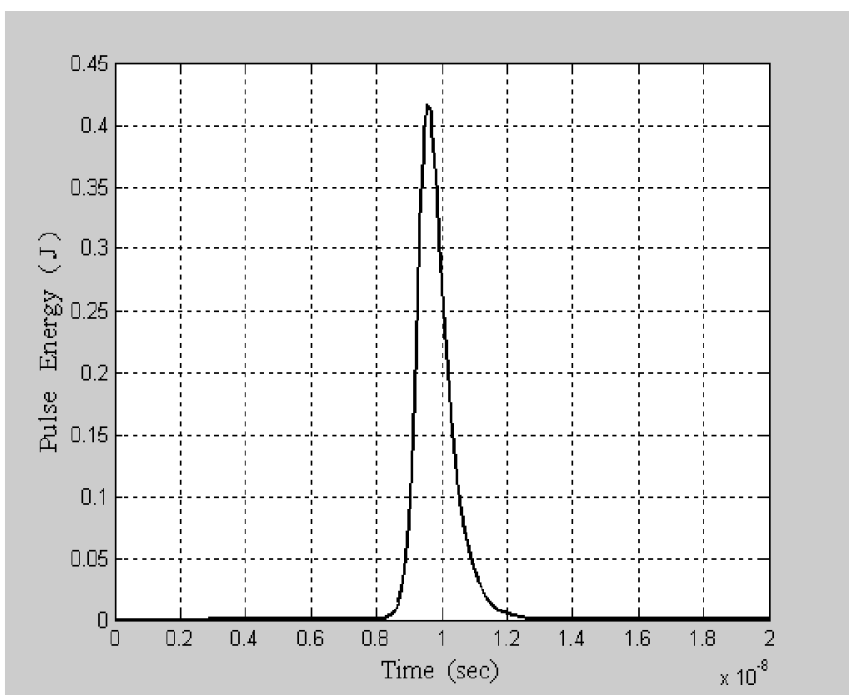

Figure 14 Development of the $Q$-switched pulse

\section{Results and analysis}

Successful implementation of the proposed simulation tool presented in this paper enabled us to investigate and analyze many parameters related to the design of solidstate lasers, as well as the ability to optimize the design to meet certain requirements. As an example, we were able to prove that at low transmission partial output coupler leads to a low laser threshold, but also possibly to poor laser efficiency if the losses due to output coupling do not dominate over other parasitic losses in the laser cavity. The partial output coupler transmission may be chosen to maximize the achieved output power, although its optimum value may be lower or higher if there are other design goals. There are optimum values for the output coupler reflectance, saturable absorber initial transmission, and the mirrors separation that maximize the output laser pulse energy and minimize the size of the laser system. Figures 15 and 16 illustrate a set of results in which the required output energy is $6 \mathrm{~J} /$ pulse. Using the proposed simulation tool we found that the optimum output coupler reflectance is $30 \%$ and the optimum mirrors separation is $13 \mathrm{~cm}$.

Another set of results obtained using the proposed simulation tool and related to the optimization of the Q-switched laser parameters to produce maximum output energy. The system is optimized using the two variables introduced in [8]. The first variable is the normalized gain factor, $\mathrm{Z}$, defined as the ratio of the logarithmic round-trip small signal gain to the nonuseful dissipative loss, $\mathrm{Z}=\left(\ln G_{o}{ }^{2} / L^{\prime}\right)=5.5$, in which $L^{\prime}$ is the round trip loss after adding the saturable absorber and is given by, $L^{\prime}=L+2 \sigma_{e s} n_{o} l_{s}$. 


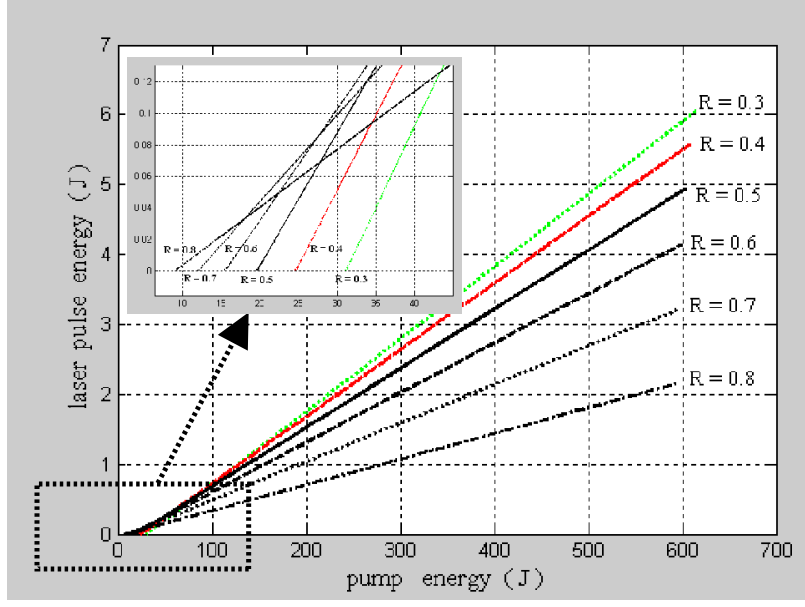

Figure 15 Output energy vs pump energy at different output coupler reflectivities

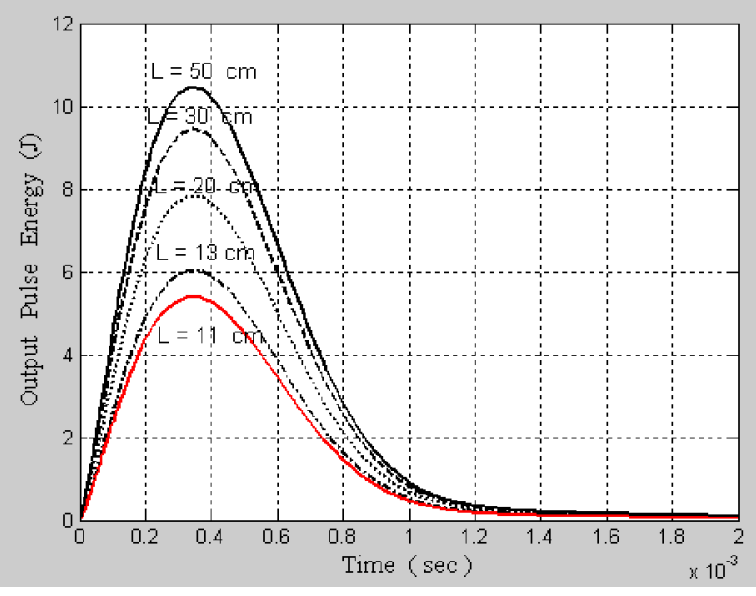

Figure 16 Changing of the output pulse energy with the change of the resonator length.

The second optimization parameter is, $b$, is determined by the ratio of the absorber cross section, $\sigma_{\mathrm{gs}}$, to the stimulated emission cross section of the gain medium, $\sigma$, and also depends on level degeneracy and relaxation rates within the laser and absorbing media, and is given by, $\left(\sigma_{\mathrm{gs}} \gamma_{\mathrm{s}} / \sigma \gamma\right)=1.34$. From [10] $\gamma_{\mathrm{s}}=1 /\left(1-\sigma_{\mathrm{es}} / \sigma_{\mathrm{gs}}\right)=1.44$ and from [4] $\gamma=1$ for four-level lasers. For $\mathrm{Z}=5.5$ and $\mathrm{b}=1.34$ the optimum coupling parameters that maximize the laser output pulse energy are $\mathrm{x}_{\mathrm{opt}}=0.8$ and $\mathrm{y}_{\mathrm{opt}}=0.68$. Using these parameters it is also found that the optimum output coupler reflectivity is $65 \%$, the unsaturated transmission of the absorber , $\mathrm{T}_{\mathrm{opt}}$, is $40 \%$, and the mirrors separation is $13 \mathrm{~cm}$. This leads to output pulse energy of $97 \mathrm{~mJ}$ with pulse width (FWHM) of 4 nsec, and peak power is approximately $25 \mathrm{MW}$. These results are shown in figures 17 and 18.

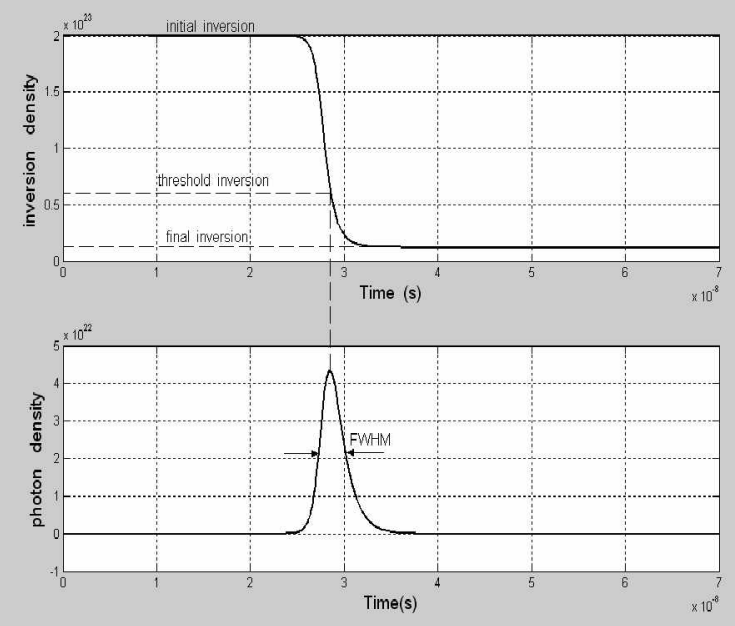

Figure 17 Time evolution of inversion and photon densities after adding the $Q$-switch.

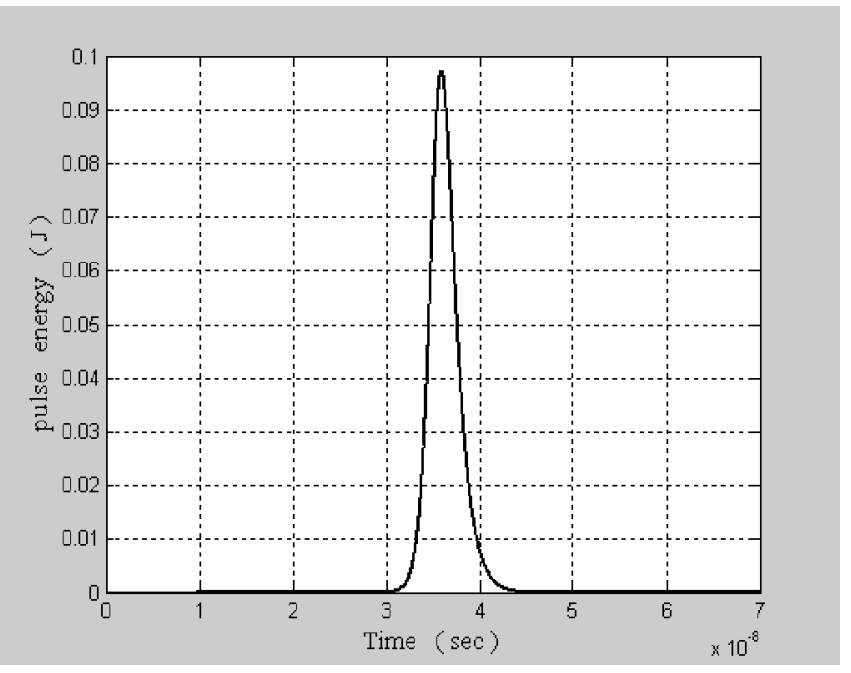

Figure 18 Development of a $Q$-switched pulse 
To show the powerful capabilities of the proposed simulator a mathematical model of a pumping circuit that consists of series connected xenon flashlamps is added to the model and solved using SIMULINK. The model is also to predict the parameters of the pulse forming network according to the required input energy for the flashlamp. Using the proposed simulator after adding the power supply model we are able to customize and optimize the overall pulsed solid-state laser system design parameters to satisfy a predetermined output energy, beam shape and geometrical dimensions. To introduce these capabilities, figure 19 illustrates the effect of varying the charging voltage on the flashlamp input energy under different pumping pulse width, $T_{P}$. Analyzing this figure, we conclude that at each $T_{P}$, there is a maximum and minimum limits of the charging voltage to be practically suitable. For example, to get $600 \mathrm{~J}$ flashlamp input energy the required charging voltage is approximately $2.2 \mathrm{KV}, 3.13 \mathrm{KV}$, or $4.5 \mathrm{KV}$ if the pumping pulse width is $900 \mu \mathrm{sec}, 300 \mu \mathrm{sec}$, or $100 \mu \mathrm{sec}$ respectively. Figure 20 illustrates the effect of varying the pump pulse energy on the total capacitance under different pumping pulse width, $\mathrm{T}_{\mathrm{P}}$. Studying figure 20 yields to conclude that the total capacitance is a nonlinear increasing function with respect to the pump energy increase for any pulse width. This conclusion is practically useful for specifying the total capacitance and relates it to the commercially available components. Figure 21 illustrate the effect of varying the charging voltage on the lamp impedance parameter, $\mathrm{K}_{\mathrm{o}}$, under different input energies. This figure shows that the lamp impedance is non-linearly increases as increasing the charging voltage in spite of the input energy. It also shows that the lamp impedance does not greatly dependent on the input energy at lower charging voltage, up to $1 \mathrm{KV}$, while the input energy is nonlinearly increases as increasing the charging voltage. The optimum limit is particularly dependable on the practical implementation and components availability.

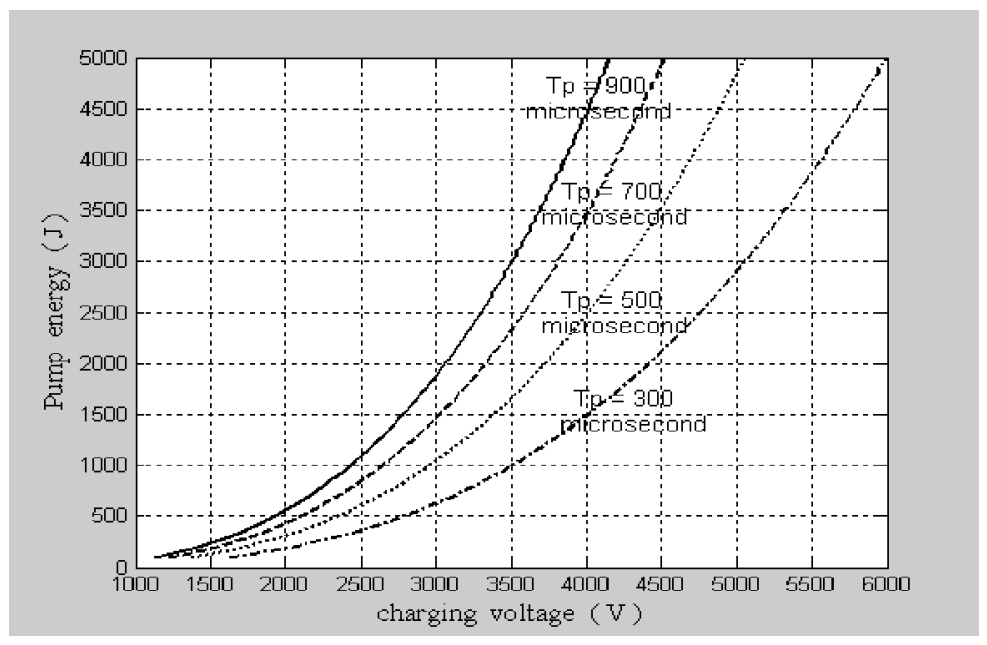

Figure 19 Flash lamp input energy vs voltage for different pumping pulse width, $T_{P}$. 


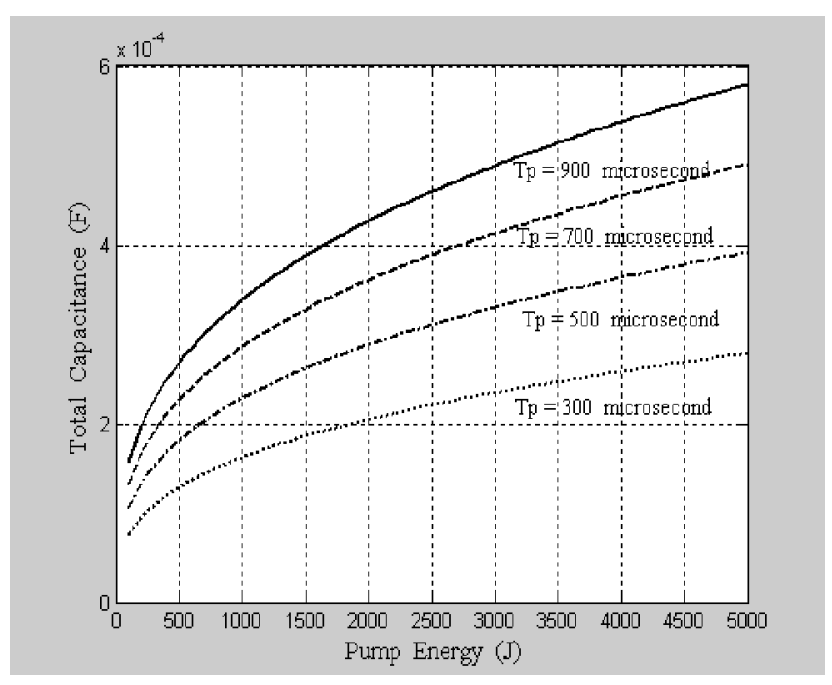

Figure20 Lamp impedance parameter $\mathrm{K}_{\mathrm{o}}$ vs charging voltage for different input Energies $E_{\text {in }}$

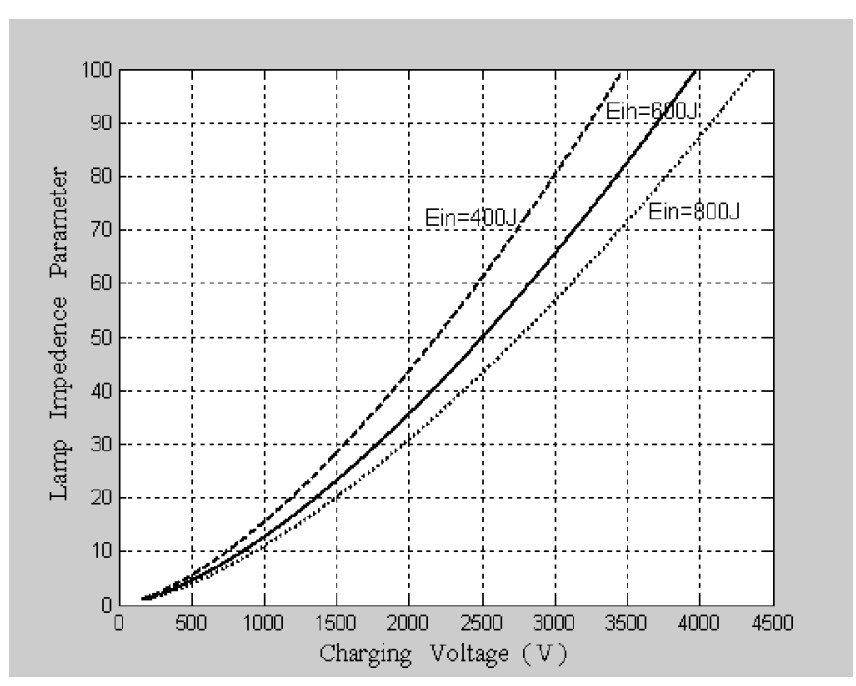

Figure 21 The charging capacitance vs pumping energy for different pump pulse width, $T_{P}$.

\section{Conclusion}

A computationally efficient model including laser intrinsic, Q-switch, flashlamp and power supply models for Nd:YAG solid-state laser simulations and design was presented. The proposed consistent model was constructed using a SIMULINK in MATLAB. The proposed model was used to predict the laser dynamic behaviors in time and frequency domains. Comparison between measurement and simulation results of some of the laser parameters under different conditions confirmed that the predicted performance using the proposed model is in good agreement with the measured and published performance. The presented results show that the proposed laser model can correctly predict the design parameters of the pulsed Nd:YAG laser systems. We anticipate that the simulation setup for pulsed Nd:YAG laser systems need to be verified and tested over a wide range of conditions to be suitable for prediction of all the design parameters under different conditions. It is deduced that the output coupler reflectance and saturable absorber initial transmission are crucial for the pulse energy and pulse width.

Using the proposed model and simulation setup many parameters such as free running output power, photons dynamics, relaxation oscillation, power spectrum and Q-switch pulse energy have been computed and graphically presented. Using the presented model system was optimized with respect to mirrors separation, output coupler reflectance and the saturable absorber initial transmission. The proposed simulator enabled us to optimize laser systems and as a demonstration of this capability, a Q-switched Nd:YAG laser was designed and optimized using the proposed simulator to maximize the output pulse energy. System optimization showed that the optimum output coupler reflectance required to achieve the required peak power is 0.65 , mirror separation is $13 \mathrm{~cm}$ and the 
saturable absorber initial transmission is $40 \%$. This leads to pulse energy of $97 \mathrm{~mJ}$ and a pulse width of $4 \mathrm{~ns}$. The proposed simulator provides the ability to change any of the laser system parameters and shows the corresponding change in the laser output or the other laser parameters. Also using the proposed simulator, analysis was made for the pumping power supply parameters under different pumping parameters. At the end, the presented results and conclusions confirm the ability of using the proposed simulation tool for the design and optimization of pulsed Nd:YAG lasers however it requires extra effort to include more practical experiences. We also are working toward enhancing the proposed simulation tool by adding an easy to use GUI.

\section{$\underline{\text { References }}$}

1. N. P. Barnes, Solid-State Lasers from an Efficiency Perspective, IEEE Journal of Selected Topics in Quantum Electronics, Vol. 13, No. 3, May/June 2007.

2. H-J Kim, et al., Active two-pulse superposition technique of a pulsed Nd:YAG laser, Optical Engineering, Vol 37, Issue 6, P. 1780-1784, 1998.

3. K-S Kim, et al., Output performance of a Nd:YAG laser with mixed operation in the CW and pulsed modes, Optical Engineering, Vol 34, Issue 5, P. 1413-1416, 1995.

4. W. Koechner, Solid-State Laser Engineering, fifth ed., Springer-Verlag, New York, Heidelberg, 2001.

5. R. Ifflainder, Solid-State Laser for Materials Processing, Springer-Verlag, New York, Heidelberg, 2001.

6. B. A. See, Mathematical modeling of an Nd:YAG laser, Optical Engineering, Vol. 33,No. 10, P. 3364-3367, October 1994.

7. J. J. Degnan, Theory of the optimally coupled Q-switched laser, IEEE J. Quant. Elec., Vol. 25, P. 214-220, 1989.

8. J. J. Degnan, Optimization of passively Q-switched lasers, IEEE J. Quant. Elec., vol. 31, P. 1890-1901, 1995.

9. L. Chen, et al., Characteristics of a passively Q-switched Nd3+:NaY(WO4)2 laser with Cr4+:YAG saturable absorber, Optics \& Laser Technology, Vol. 34, P. 347350, 2002.

10. G. Xiao and M. Bass, A Generalized Model for Passively Q-Switched Lasers Including Excited State Absorption in the Saturable Absorber, IEEE J. Quant. Elec., Vol. 33, P. 41-44, 1997.

11. Y. F. Chen, Y. P. Lan, and H. L. Chang, Analytical Model for Design Criteria of Passively Q-Switched Lasers, IEEE J. Quant. Elec., Vol. 37, No. 3, P. 462-468, March 2001.

12. J. F. Holzrichter and J. L. Emmett, Design and Analysis of a High Brightness Axial Flash Lamp, Applied Optics, Vol 8, Issue 7, P. 1459-1464, 1969.

13. A. J. Alfrey, Flashlamp-pumped dye laser as an undergraduate lab project, Am. J. Phys, Vol 49, Issue 8, P. 776-781, 1981. 
14. W-Y Kim, et al, A simple pulsed Nd:YAG laser power supply adopted ZCC method, ISIE, P. 942-945, 2001.

15. J-H Hong, et al., Long pulse generation technology of solid-state laser adopting a new real time multi-discharge method, Optics \& Laser Technology, Vol. 34, P. 203 207, 2002.

16. B. N. Upadhaya, et al., Beam quality considerations of high power Nd:YAG lasers, Optics\&Laser Technology, Vol. 34, P. 193-197, 2002.

17. D. Findlay and R. A. Clay, The Measurement Of Internal Losses In 4-Level Lasers, Physics Letters, Vol. 20, P. 277-279, 1966.

18. EG \&G Electro-Optics Corp., Flashlamp Applications Manual, 1982.

19. A. Yariv, Introduction to Optical Electronics, 2nd ed., Holt, Rinehart and Winston, New York, 1976.

20. Y. Shimony, Z. Burshtein, and Y. Kalisky , Cr4+:YAG as Passive Q-Switch and Brewster Plate in a Pulsed Nd:YAG Laser, IEEE J. Quant. Elec., Vol. 31, No. 10, P. 1738-1741, October 1995.

\section{$\underline{\text { Nomenclatures }}$}

\begin{tabular}{|c|c|l|}
\hline Symbol & Value \&Units & \multicolumn{1}{|c|}{ Description } \\
\hline $\mathrm{n}_{2}$ & Atoms $/ \mathrm{m}^{3}$ & population density of the upper laser level \\
\hline $\mathrm{n}_{1}$ & Atoms $/ \mathrm{m}^{3}$ & population density of the lower laser level \\
\hline$\Phi$ & Photons $/ \mathrm{m}^{3}$ & photon density \\
\hline$\tau_{\mathrm{f}}$ & $230 \mu \mathrm{sec}$ & upper laser level life time \\
\hline$\sigma$ & $6.5 \times 10^{23} \mathrm{~m}^{2}$ & stimulated emission cross section area \\
\hline $\mathrm{c}$ & $3 \times 10^{8} \mathrm{~m} / \mathrm{s}$ & Velocity of light \\
\hline Symbol & Value \&Units & \\
\hline$\tau_{10}$ & $\mathrm{sec}$ & $\begin{array}{l}\text { Relaxation time between lower laser level and the ground } \\
\text { level }\end{array}$ \\
\hline$\tau_{\mathrm{c}}$ & $\mathrm{sec}$ & Decay time for photons in the optical resonator \\
\hline $\mathrm{g}_{2} / \mathrm{g}_{1}$ & 1 & Degeneracy factor \\
\hline $\mathrm{L}$ & - & Combined loss per round trip \\
\hline $\mathrm{R}_{1}$ & - & Output coupler reflectance \\
\hline $\mathrm{K}$ & $\mathrm{J}^{-1}$ & Pumping coefficient \\
\hline $\mathrm{g}_{\mathrm{o}}$ & $\mathrm{cm}$ & Small signal gain \\
\hline $\mathrm{G}_{\mathrm{o}}$ & 3.74 & Single pass gain \\
\hline $\mathrm{E}_{\mathrm{th}}$ & $\mathrm{J}$ & Threshold pump energy \\
\hline $\mathrm{E}_{\mathrm{out}}$ & $\mathrm{J}$ & Output pulse energy \\
\hline $\mathrm{L}$ & $\mu \mathrm{H}$ & Total inductance \\
\hline $\mathrm{C}$ & $\mu \mathrm{F}$ & Total capacitance \\
\hline$\alpha$ & - & Damping factor \\
\hline $\mathrm{PFN}$ & - & Pulse forming network \\
\hline $\mathrm{T}_{\mathrm{p}}$ & $\mu \mathrm{sec}$ & Pump pulse width \\
\hline & & \\
\hline
\end{tabular}




\begin{tabular}{|c|c|l|}
\hline $\mathrm{K}_{\mathrm{o}}$ & $\Omega \mathrm{A}^{1 / 2}$ & Impedance parameter \\
\hline $\mathrm{fft}$ & - & Fast Fourier transform \\
\hline $\mathrm{I}_{\mathrm{s}}$ & $\mathrm{mm}$ & Saturable absorber crystal thickness \\
\hline $\mathrm{T}_{\mathrm{o}}$ & - & Small signal transmission \\
\hline$\sigma_{\mathrm{es}}$ & $2.2 \times 10^{-19} \mathrm{~cm}^{2}$ & Excited state absorption cross section \\
\hline$\sigma_{\mathrm{gs}}$ & $8.7 \times 10^{-19} \mathrm{~cm}^{2}$ & Ground state absorption cross section \\
\hline $\mathrm{N}_{\mathrm{o}}$ & $3.187 \times 10^{24} / \mathrm{m}^{3}$ & Atoms concentration in saturable absorber \\
\hline$L^{\prime}$ & - & Total loss in the resonator after adding saturable absorber \\
\hline $\mathrm{Z}$ & - & Normalized gain factor \\
\hline $\mathrm{x}_{\mathrm{opt}} \&$ & - & Passive Q-switch Optimum coupling parameters \\
\hline $\mathrm{y}_{\mathrm{opt}}$ & - & Optimum unsaturated transmission of the absorber \\
\hline $\mathrm{T}_{\mathrm{opt}}$ & $\mathrm{mJ}$ & Maximum output pulse energy \\
\hline $\mathrm{E}_{\mathrm{max}}$ & $\mathrm{nsec}$ & Q-switch pulse width \\
\hline $\mathrm{t}_{\mathrm{p}}$ & $\mathrm{nsec}$ & Round trip time \\
\hline $\mathrm{t}_{\mathrm{r}}$ & $\mathrm{cm}$ & Laser rod cross section area \\
\hline $\mathrm{A}$ & - & Q-Switch optimization factor \\
\hline $\mathrm{b}$ & - & Optical spectrum analyzer \\
\hline $\mathrm{OSA}$ & - & Pump rate \\
\hline $\mathrm{W}_{\mathrm{p}}$ & - & Graphical user interface \\
\hline $\mathrm{GUI}$ & Atoms $/ \mathrm{m}^{3}$ & Total number of atoms \\
\hline $\mathrm{n}_{\mathrm{o}}$ & - & Initial inversion \\
\hline $\mathrm{n}_{\mathrm{i}}$ & - & Inversion reduction factor \\
\hline$\gamma$ & - & $\begin{array}{l}\text { Effect of the absorption of a single photon by the saturable } \\
\text { absorber on the absorber population density. }\end{array}$ \\
\hline$\gamma_{\mathrm{s}}$ & & \\
\hline & & \\
\hline
\end{tabular}

